\title{
Paranoid Hostility but Not Depressive Symptoms Associated with Cytokines During the Premenstrual Phase in Young Women
}

\author{
Syros Ioannis, ${ }^{1,2 *}$ Gourna Chryssoula, ${ }^{3}$ Papageorgiou Charalambos,${ }^{4}$ Kolaitis Gerasimos, ${ }^{1}$ Liapi Charis ${ }^{3}$ \\ ${ }^{1}$ Department of Child Psychiatry, School of Medicine, National and Kapodistrian University of Athens, Greece \\ ${ }^{2}$ Child and Adolescent Psychiatry Unit, "Sotiria" General Hospital, Athens, Greece \\ ${ }^{3}$ Department of Pharmacology, Medical School, University of Athens \\ ${ }^{4}$ Department of Psychiatry, University of Athens, Eginition Hospital
}

\begin{abstract}
It is well known that some women, during their premenstrual phase, express Hostility (Ho) and Depressive symptoms (De). Various studies indicate that Ho and De correlate with circulating concentrations of cytokines. These data support the hypothesis of an inflammation-sensitive mechanism linked to certain psychological states. The aim of this study was to correlate Ho and De with selective proinflammatory cytokines during the menstrual cycle in young women. Twenty-two physically healthy medical students, mean age 22.95+2.83(SD), were studied. All reported regular menses that ranged between 27-33 days. Blood was drawn three times across a single cycle: at follicular phase (FL), at mid luteal phase (ML) and at late luteal phase (LL) corresponding to the premenstrual phase. The following proinflammatory cytokines were determined at each sample: Interleukin 8 (IL-8), Interleukin 6 (IL-6), Interleukin $1 \alpha$ (IL-1 $\alpha$ ), and Tumor Necrosis Factor- $\alpha$ (TNF- $\alpha$ ). Immediately before blood sampling the women completed the Zung self-rating depression scale and the hostility and direction of hostility questionnaire (HDHQ), which comprises five subscales: urge to act out hostility, criticism of others, paranoid hostility, self-criticism and delusional guilt. Spearman's rank correlation was used to determine the relation between variables at the specific time points; $\rho<0.05$ was considered significant. Mean values of cytokines did not change significantly throughout the menstrual cycle. Paranoid hostility was positively correlated with IL-8 (Rs=0.476, $p=0.046)$, IL- $1 \alpha(R s=0.458, p=0.048)$ and TNF- $\alpha$ ( $R s=0.518, p=0.023$ ) in LLphase. No other significant correlation was found. Cytokines correlate with paranoid hostility and this correlation is probably related with the premenstrual hormonal changes. It is difficult to evaluate the clinical significance of our findings, nevertheless, when exploring the above association, it is necessary to take into consideration the phase of the cycle as well as the special aspects of Ho we seek to study.
\end{abstract}

Keywords: Hostility, Depression, Depressive symptoms, Cytokines, Menstrual cycle, Correlation

Abbreviations: HO: Hostility; DE: Depressive symptoms; IL-8: Interleukin 8; IL-6: Interleukin 6; IL-1 $\alpha$ :Interleukin $1 \alpha$; TNF- $\alpha$ :Tumour Necrosis Factor- $\alpha$; HDHQ: Hostility and Direction of Hostility Questionnaire; FL: Follicular Phase; ML: Mid Luteal Phase; LL:Late Luteal Phase

\section{Introduction}

Ho is a multifaceted psychological construct comprising cognitive, affective and behavioural dimensions, while it is thought to be a potentially 'toxic' characteristic of the type A personality. In addition, Depression is a serious illness with a high incidence in the general population ${ }^{1}$ It is associated with an increased risk of

suicide, other comorbid emotional and behavioural disorders, development of metabolic abnormalities, and generally increased allcause mortality. Both psychological conditions have been related to premature mortality as well as to increased incidence of Coronary heart disease? ?-5 $^{2-5}$
Quick Response Code:

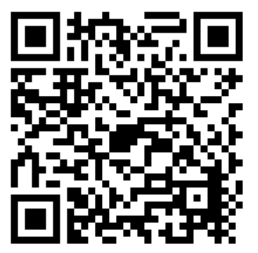

*Corresponding author: Syros Ioannis, Department of Child Psychiatry, School of Medicine, National and Kapodistrian University of Athens, "Aghia Sophia" Children's Hospital, Athens, Greece

Received: 13 April, 2021

Published: 12 May, 2021

Citation: Syros I, Gourna C, Papageorgiou C, Kolaitis G, Liapi C. Paranoid Hostility but Not Depressive Symptoms Associated with Cytokines During the Premenstrual Phase in Young Women. SOJ Neuro Neurosci. 2021;1(1):1-7. DOI: 10.53902/SOJNN.2021.01.000505 
On the other hand, cytokines are a broad category of small proteins especially important in the immune system, while they modulate the balance between humoral and cell-based immune responses, and they regulate the maturation, growth, and responsiveness of particular cell populations. The primary role of cytokine TNF- $\alpha$ is in the regulation of immune cells. If macrophages detect an infection, they release TNF- $\alpha$ in order to alert other cells of the immune system as well as cells of other tissues, leading to inflammation. TNF- $\alpha$ is activated by IL- $1 \alpha$ which is also a cytokine produced mainly by activated macrophages, and is responsible for the production of inflammation. Likewise, IL-6 is a cytokine which stimulates the inflammatory and autoimmune processes in many neurological diseases. IL-8 is also one of the major mediators of the inflammatory response; it is secreted by several cell types and functions as a chemo attractant and as a potent angiogenic factor. ${ }^{6}$

Elevated activity of the cytokines reported above have been implicated in a variety of human diseases including clinical depression..$^{7-18}$ However, few studies where up to middle-aged individuals participated, and which used valid psychometric tools did not detect an association. ${ }^{19,20}$ On the other hand, several studies comprising younger women ${ }^{21-23}$ as well as other special populations. ${ }^{13,24-26}$, reportedthe positive association between various cytokines and Ho. For example, Suarez et $\mathrm{al}^{23}$ detected a positive correlation between Il $-1 \alpha$, Il-8 and Ho in a sample of healthy young women, while,likewise, in a sample of agitated psychotic patients Ho was positively associated with TNF- $\alpha{ }^{24}$

These data support the hypothesis of a likely inflammation-sensitive mechanism linked to certain psychological states.27However, inconsistent results have emerged, suggesting that the sex and age of the samples, ${ }^{21}$ thecoexistence of De, ${ }^{14,28}$ other cardiovascular risk factors, as well as the medical co morbidity ${ }^{29-31}$ may influence the relation above.

The aim of this study was to correlate Ho and De with various proinflammatory cytokines (IL-8, IL-6, IL- $1 \alpha$, TNF- $\alpha$ ) during the menstrual cycle in physically healthy young women. During the cycle, significant fluctuations in reproductive hormones are reported. Specifically, estradiol and progesterone are increased in the MLphase, compared to the FLphase, while subsequently, physical and psychological distress symptoms manifest in the context of premenstrual syndrome (PMS), including depressed mood, anger and irritability. The prevalence of reproductive age women affected with PMS worldwide amounts to $47.8 \%$; about $20 \%$ of these women experience symptoms severe enough to disrupt their daily activities. ${ }^{32}$ In addition, comparative differences in progesterone and estradiol fluctuations appear to occur between the ML andLL phase, indicating that these relative differences within the same phase may mediate in the correlations studied in this study. ${ }^{33}$ Taking into account the above, hormone fluctuations throughout the cycle could serve as an interesting model in nature to explore these relationships and increase our understanding of the biological pathways that may mediate these links. The hypotheses to be tested were that: (1) there will be a positive correlation between cytokines concentrations, De and Ho scores. (2) There will be a marked effect of the menstrual cycle phase regarding the association between De, Ho and the tested cytokines.

\section{Material and Methods}

This study was part of a larger investigation we conducted on the relation between psychometric and biochemical factors across the menstrual cycle in healthy young women. ${ }^{34,35}$ Blood sampling and interviews were conducted during the period January 1, 2003 to December 31. 2004.

\section{a) Participants}

Twenty-two healthymedical students, mean age 22.95+2.83(SD) years old, were studied, after exclusion from the analysis of 2 women with missed data. The women received written information on the aim and procedures of the study and gave their informed consent. All of them were interviewed and examined by an internist. All women were physically healthy, with no clinical evidence of cardiovascular disease, autoimmune disorders etc. and were not using medication (i.e., hormonal contraceptives, Selective Serotonin Reuptake Inhibitors, anti-inflammatory agents etc.); they reported regular menstruation ranging between 27 and 33 days and had no signs of acne or hirsutism. Clinical examinations included weight, height, blood pressure and electrocardiogram. The Body Mass Index (BMI) was calculated as weight $/$ height $^{2}\left(\mathrm{~kg} / \mathrm{m}^{2}\right)$. On the days of blood sampling, women were asked about possible symptoms of inflammation or illness, in order to rule out this case that would affect the measured cytokines. In addition, the women did not report signs of significant psychiatric symptoms (clinical depression or any other mental disorder). The diagnosis of a psychiatric disorder was made by a short psychiatric interview and observation by the internist, without the assistance of a specialized psychiatric interview or test. In addition, women were asked to record their premenstrual symptoms using a simple self-report questionnaire. All procedures followed were in line with the ethical standards of the responsible committee on human experimentation (institutional and national) and with the 1975 Helsinki Declaration, as revised in 2008.

\section{b) Cytokinesmeasurements}

Venous blood samples were drawn between 8-10am, after overnight fasting for a minimum of 12 hours, three times at each cycle: at the FL phase, ML phase, as well as the LL phase, which corresponds to the premenstrual phase. Cycle phases were tracked based on participants' self-report of the onset of the last menses. ML and LL phase measurements were recorded at +21 day and +28 day from the onset of the last menses, respectively. The proinflammatory cytokines below were identified at each sample: IL-8, IL-6, IL- $1 \alpha$, and TNF- $\alpha$.

\section{c) Assessment of depressive symptoms and hostility}

Depressive symptomatology was assessed by the greek version of Zung Self-Rating Depression Scale, a 20-item self-report questionnaire, which is widely used as a screening tool covering affective, psychological and somatic symptoms related to depression..$^{36,37}$ The questionnaire takes about 10 minutes to complete and items 
are formulated in terms of positive and negative statements. Each item is rated on a likert scale ranging from 1 to 4 . An overall score is obtained by summing the individual item scores and ranges from 25 to 80 . The scores provide indicative ranges for depression severity that can be useful for clinical and research purposes, but the Zung scale cannot replace a comprehensive clinical interview for the diagnosis of depression.

Individuals also completed the Hostility and Direction of Hostility Questionnaire (HDHQ) ${ }^{38}$ This assessment instrument represents a measure of a wide range of hostility manifestations as a personality trait that reflects the subject's response to frustrating occurrences, and consists of five subscales: (a) the urge to act out hostility, (b) criticism of others, (c) paranoid (or projected delusional) hostility, (d) self-criticism, and (e) delusional guilt. Primary three subscales are summed to make an extra-punitive score, and the other two are summed to yield an intropunitive score. Each subscale score is ranged from 0 to 11, therefore the total Hostility score ranges from 0 to 55 by summing the five subscale scores. HDHQ has been widely used in Greek community and clinical samples. ${ }^{39}$ Participants completed these questionnaires immediately before blood sampling at each phase of the cycle.

\section{d) Statistical analysis}

Data are presented as mean \pm SD. The relations between variables at each phase of the menstrual cycle were detected using the Pearson coefficient of correlation (R). The Spearman's rank correlation coefficient (RS) was also computed when normality assumption was not satisfied. All reported $\rho$-values were considered significant when less than 0.05 . Comparisons within cycle phases were performed by the one-way analysis of variance (ANOVA) for repeated measures followed by Bonferroni correction for multiple comparisons. When data were not normally distributed, the non-parametric Friedman test was used for comparisons within phases and also the Wilcoxon Signed Ranks test for paired comparisons. All reported $\rho$-values were considered significant when less than 0.05 .

\section{Results}

Mean valuesof cytokines did not change significantly throughout the menstrual cycle, except for TNF- $\alpha$ which from $7.14 \mathrm{U} / \mathrm{ml}$ in ML phase rose to $12.14 \mathrm{U} / \mathrm{ml}$ in LL phase (Wilcoxon signed ranks test, $\mathrm{p}=0.016$ ) (Table 1$)$

Table 1: Mean values $\pm S D$ of the Cytokines and Psychometric scores measured in the medical students in the F phase, ML phase, and LLphase.

\begin{tabular}{|c|c|c|}
\hline Parameter & Mean U/ml & Std. Dev. \\
\hline \multicolumn{3}{|l|}{ IL-8 } \\
\hline phase F & 26,11 & 7,32 \\
\hline phase ML & 24,98 & 5,43 \\
\hline phase LL & 30,31 & 13,09 \\
\hline \multicolumn{3}{|l|}{ IL-6 } \\
\hline phase F & 9,45 & 4,71 \\
\hline phase ML & 9,09 & 3,95 \\
\hline phase LL & 9,88 & 5,88 \\
\hline \multicolumn{3}{|l|}{ IL-1 $\alpha$} \\
\hline phase F & 5,70 & 3,10 \\
\hline phase ML & 6,59 & 3,46 \\
\hline phase LL & 7,30 & 6,12 \\
\hline \multicolumn{3}{|l|}{ TNF- $\alpha$} \\
\hline phase $\mathrm{F}$ & 9,23 & 9,64 \\
\hline phase ML & 7,14 & 4,77 \\
\hline phase LL & 12,14 & 13,10 \\
\hline \multicolumn{3}{|c|}{ Depression (Zung self-rating scale scores) } \\
\hline phase F & 47.69 & 8.28 \\
\hline phase ML & 46.57 & 8.96 \\
\hline phase LL & 46.16 & 9.68 \\
\hline \multicolumn{3}{|c|}{ Hostility (Hostility and Direction of Hostility Questionnaire scores) } \\
\hline \multicolumn{3}{|c|}{ Paranoid Hostility } \\
\hline phase F & 1.76 & 1.37 \\
\hline phase ML & 1.93 & 1.35 \\
\hline phase LL & 1.58 & 1.42 \\
\hline
\end{tabular}


Table 2: Statistically significant correlations between Hostility and Cytokines $(a=5 \%)$ in the various phases of cycle (the phase marked denotes the significant correlation)..

\begin{tabular}{|c|c|c|c|c|c|}
\hline \multirow{2}{*}{ Psychometric Parameter } & \multirow{2}{*}{ Cytokines } & \multicolumn{3}{|c|}{ Cycle Phase } & \multirow{2}{*}{ Rs (p-value) } \\
\hline & & Follicular & Mid Luteal & Late Luteal & \\
\hline \multirow{6}{*}{ HDHQ Paranoid Hostility } & \multirow{2}{*}{ IL-8 } & & & \multirow{2}{*}{+} & 0.476 \\
\hline & & & & & $(0.046)$ \\
\hline & \multirow{2}{*}{ IL-1 $\alpha$} & & & \multirow{2}{*}{+} & 0.458 \\
\hline & & & & & $(0.048)$ \\
\hline & \multirow{2}{*}{ TNF- $\alpha$} & & & \multirow{2}{*}{+} & 0.518 \\
\hline & & & & & $(0.023)$ \\
\hline
\end{tabular}

Paranoid hostility was positively correlated with IL-8(Rs=0.476, $\mathrm{p}=0.046), \quad \mathrm{IL}-1 \alpha \quad \mathrm{Rs}=0.458, \mathrm{p}=0.048)$ and TNF- $\alpha \quad(\mathrm{Rs}=0.518$, $\mathrm{p}=0.023$ ) in the LL phase. (Table 2)

No significant correlation was found between the other ingredients of Ho, Zung scores and cytokines in LL phase, while no correlation was detected in FL and ML phases.

\section{Discussion}

Surprisingly, in our study, we did not detect any correlation between De and proinflammatory cytokines during the menstrual cycle. Taking into account that cytokines are influenced by various traditional risk factors (eg BMI, hypertension, hyperlipidemia, smoking), ${ }^{30,31}$ we speculate that in our study sample of physically healthy young women, the possibilities of confounding by the factors above are quite few. Most studies detect a positive association between De and cytokines. However, these studies vary in size and sample characteristics.

More specifically, in two studies by Suarez et al. ${ }^{23,13}$ an association was detected between De, IL-1 $\alpha$, TNF- and IL-8, in two samples with many common characteristics to ours (healthy, non-smoking, premenopausal women, aged 23-49 years, as well as apparently healthy men, with similar age range, 18-43 years, respectively). Nevertheless, the investigators used a different questionnaire to assess De (Beck Depression Inventory) and also the tested cytokines were assessed after in vitro lip polysaccharide (LPS) stimulation of undiluted whole blood. In addition, in the female sample, data were only collected during the FL of the cycle.

In accordance to the studies above, Miller GE et $\mathrm{al}^{15}$ showed a positive correlation between IL-6, TNF- $\alpha$ and BDI score. The one hundred participants were primarily women on average older than our sample. In the Multi-ethnic study of Atherosclerosis where a large sample of healthy adults recruited multiple regression analysis revealed a positive association between De and IL-6, but the association emerged only at high scores detected by the self-report scale (CES-D higher than 21). ${ }^{10}$ Two other studies have also found an association between IL-6, TNF- $\alpha$ and De, but included older persons. ${ }^{16,18}$

On the contrary, some studies describe, similarly to our work, no relation between De and cytokines. Thus, in a population sample of healthy middle-aged women (47-59 years old), drawn from the whitefall II epidemiological cohort, the investigators did not manage to detect an association between De and cytokines IL-6 and TNF- $\alpha .{ }^{19}$ General Health Questionnaire-30 was used to define Depression. Similarly, no correlation was found in another study where only 12 depressed outpatients (aged 19-45 years) participated. The authors used Hamilton Rating Scale for Depression and the Profile of Mood States in order to detect De. ${ }^{20}$

Contradictory results could be attributed to the fact that factors such as age, cardiovascular risk factors, medical co morbidity and treatment, obesity as well as sleep quality of the individuals may differ in the various study samples. In addition, chronic exposure to stress also appears to interfere with the above relationship; ${ }^{40,41}$ we assume that in our sample of healthy young women, the exposure to stress experiences was shorter, compared to the duration of a sample of older participants. Thus, the different results compared to our study could also be attributed to the different age of the study samples. In addition, the above studies indicate that the correlation between these two variables may be influenced by whether the studied samples included clinically depressed individuals or individuals who had some symptoms only (below the diagnostic threshold for depression). In the assessment of psychometric properties of Greek version of Zung rating scale, authors concluded that the cut-off point $44 / 45$, which corresponded to the $90^{\text {th }}$ percentile of the studied sample, could be used as a screening limit for further evaluation of clinical depression. ${ }^{36}$ On the other hand, in our sample, young women scored slightly higher than the cut-off point in De. Nevertheless, brief psychiatric examination by the internist did not reveal signs of a clinical depression.

On the contrary, we detected a significant correlation between IL- $1 \alpha$, IL- 8 and TNF- $\alpha$ with the paranoid aspect of HDHQ in the premenstrual phase. Ho is a multifaceted psychological construct. Paranoid Hostility is considered to be a cognitive and extroverted ingredient of Ho, as well as a consequence of paranoid thoughts. ${ }^{42}$ The studies so far that examine the relationship of Ho with cytokines are generally scarce, and to our knowledge, this is the first research attempted to test this kind of association throughout the menstrual cycle. In addition, the positive correlation found between paranoid aspect of Ho and the cytokines under study may raise questions about whether this is a novel finding. In particular, in the study by Larsen et al, ${ }^{24}$ it was found that in a group of agitated patients, both those suffering from schizophrenia, as well as the general psychiatric population, who admitted to an acute psychiatric inpatient department, Ho, as measured by the positive and Negative Syndrome Scale, Excited Component, was positively correlated with the cytokine TNF- $\alpha$. This finding, combined with the results of our study, 
raises the question of whether the psychotic aspects of Ho, driven by delusional thoughts, are those that are in specific association with some cytokines, and especially with TNF- $\alpha$, and not all aspects of Ho. Furthermore, Boisclair Demarble et $\mathrm{al}^{22}$ examining the relationship between total Ho and various cytokines in a sample of 118 women, aged 20-64years, using a different questionnaire to assess Ho(Cook-Medley Hostility Inventory), found a positive correlation, mainly in younger women.Suarez et $\mathrm{al}^{23}$ similarly to our research, detected a correlation between Ho scores with IL-1 $\alpha$ and IL-8, but in the FL phase of the menstrual cycle; to note that the investigators also used Cook-Medley inventory. Furthermore, as we previously reported, the sample consisted of 44 healthy non-smoking premenopausal women, aged 23-49 years, the data were collected during one phase of the menstrual cycle and thus, the associations, regarding the other phases, have not been investigated; finally, the cytokines were assessed after LPS stimulation.The same authors, in a previous studytwo years earlier, had shown a positive association between Ho and LPS stimulated TNF- $\alpha$ in a sample comprising healthy non-smoking men. ${ }^{26}$ Finally, as concerns the relation between IL- 6 and Ho, in a prospective study where older adults under chronic stress participated, no correlation was found, albeit the age of the individuals could influence the association. ${ }^{29}$

Extensive investigation has focusedon the neuroendocrine and neurochemical mechanisms which interfere with the link between psychological states and cytokines system. ${ }^{40,43}$ In regards to the association between Ho and TNF- $\alpha$, it has been speculated that stimulation of adrenergic receptors via norepinephrine may play a regulatory role. Furthermore, serotonin deficiency has also been reported as a possible mechanism which links TNF- $\alpha$ with hostile and aggressive behavior. ${ }^{26}$

We could also hypothesize that in the positive association between Ho and cytokines, low density lipoprotein (LDL) levels might interfere. ${ }^{15}$ Speculation is based on the observation that in some studies, hostile and aggressive subjects express elevated levels of LDL cholesterol ${ }^{44,45}$ as well as on theories which support that oxidative modified LDL lipoproteins might expand the inflammatory response. ${ }^{15,46,47}$ This process may not be expressed in depressed subjects while various studies indicate a negative correlation between LDL and Depression.48 This state is compatible with our findings which did not reveal a relationship between De and proinflammatory cytokines levels. Regardless of the mechanism(s) responsible, the unlike pattern of findings acquired in our study propose that De and Ho may induce the cytokines expression via different pathways. Some authors demonstrated a joint effect of Ho and depression on the plasma concentrations of cytokines highlighting the importance of considering both the independent and interactive relationships among these psychological factors in the definition of this association. ${ }^{14,15,23,28}$ It's obvious that due to the nature of this study, it's difficult to establish the directionality of these correlations.

\section{Limitations}

Findings of this study should be interpreted with caution because of several limitations. The reliance on a self-report test to assess the presence of De and Ho traits should be considered with caution; nevertheless, self-reports are widely used in epidemiological studies and are generally considered satisfactory tools to detect and assess depression and Ho.,49 Additionally, while the Zung Scale cannot substitute a comprehensive clinical interview, no formal structured interview was used to establish the absence of a psychiatric disorder (e.g., through the MINI-plus, CIDI or SCID). In contrast, psychiatric assessment of participants was based on their personal reference as well as on the brief psychiatric history taken by the internist. Moreover, the sample size was relatively small, albeit it consisted of youths without remarkable confounding factors such as medication, physical co-morbidity, known cardiovascular risk factors etc. Finally, another limitation of this study was that fluctuations of the cytokines were measured for only a month, while due to the cross-sectional design of this research; we can simply no causal relation between the parameters examined.

\section{Conclusion}

In the LL phase, proinflammatory cytokines are associated with paranoid hostility, an extra punitive aspect of Ho. The clinical significance of the present findings is difficult to estimate, but we can speculate that the relation between Ho and cytokines appear to be influenced by the special aspect of Hostility we seek to study. We also assume that the phases of the cycle need to be taken into consideration when examining the relation above. Therefore, during the LL phase, the distress experienced by young women, with the parallel mobilization of stress hormones (norepinephrine, cortisol), and, at the same time, the decline curves of estrogen and progesterone, may play interactively a regulatory role in the positive correlation between proinflammatory cytokines and paranoid hostility. In other terms, the increased vulnerability to stress experienced in women during LL period,,$^{50}$ probably due to the influence of gonadal hormones to amygdala, ${ }^{51,52}$ might interfere with the positive correlation between cytokines and paranoid hostility. On the other hand, selected cytokines in our study appear to interact with this particular component of Ho, thus showing the reason why some studies reveal a correlation between the two variables, while others do not. Possibly, the reason is that different studies record hostility through different tools, which are designed to record different aspects of this personality trait.

On the other hand, we failed to detect a significant correlation between De and cytokines across the menstrual cycle; however, our results are partially compatible with the literature so far. Future research including extended study periods as well as larger sample size groups is needed in order to define the causality between the psychological factors and the cytokines we choose to study, as well as the biological pathways which mediate in this association.

\section{Acknowledgments}

None.

\section{Fundings}

This research did not receive any specific grant from funding agencies in the public, commercial, or not-for-profit sectors. 


\section{Conflict of Interests}

The authors state that there are no conflicts of interest regarding the publication of this paper.

\section{References}

1. Van Reedt Dortland AK, Giltay EJ, Van Veen T, et al. Associations between serum lipids and major depressive disorder: results from the Netherlands Study of Depression and Anxiety (NESDA). J Clin Psychiatry. 2010;71(6):729-736.

2. Assari S. Hostility, Anger, and Cardiovascular mortality among black and whites. Rec Cardiovasc med. 2017;6(1):e34029.

3. Igna CV, Julkunen J, Vanhanen $\mathrm{H}$, et al. Depressive symptoms and serum lipid fractions in middle-aged men: physiologic and health behavior links. Psychosom Med. 2008;70(9):960-966.

4. Miller TQ Smith TW, Turner CW, et al. A meta-analytic review of research on hostility and physical Health. Psychol Bull. 1996;119(2):322-348.

5. Barefoot JC, Grantdahlstrom W, Williams RB. Hostility, CHD incidence, and total mortality: A 25-year folow-up study of 255 Physicians. Psychosom Med.1983;45(1):59-63.

6. Cytokine. Stedman's Medical Dictionary $\left(28^{\text {th }}\right.$ ed $)$. Wolters Kluwer Health, Lippincott Williams \& Wilkins; 2013.

7. Talarowska M, Blizniewska K, Szemraj J, et al. Personality traits as a possible factor in the inflammatory response in the first depression episode and in recurrent depressive disorders. The European Journal of Psychiatry. 2018;32(2):63-71.

8. Dahl J, Ormstad H, Hans Christian D, et al. The plasma levels of various cytokines are increased during ongoing depression and are reduced to normal levels after recovery. Psychoneuroendocrinology. 2014;45:77-86.

9. Dowlati Y, Herrmann N, Swardfager W, et al. A meta-analysis of cytokines in major depression. Biol Psychiatry. 2010;67(5):446-457.

10. Ranjit N, Diez-Roux AV, Shea S, et al. Psychosocial factors and inflammation in the Multi-Ethnic Study of theroschlerosis. Arch Int Med. 2007;167(2):174-181.

11. Schiepers JG, Wichers MC, Maes M. Cytokines and major depression. Progress in Neuro-Psychopharmacology and Biological Psychiatry. 2005;29(2):201-217.

12. O'Brien SM, Scott LV, Dinan TG. Cytokines: abnormalities in major depression and implications for pharmacological treatment. Hum Psychopharmacol. 2004;19(6):397-403.

13. Suarez EC, Krishnan RR, Lewis JG. The relation of severity of depressive symptoms to monocyte-associated proinflammatory cytokines and chemokines in apparently healthy men. Psychosom med. 2003;65(3):362-368.

14. Suarez EC. Joint effects of hostility and severity of depressive symptoms on plasma Interleukin-6 concentration. Psychosom med. 2003;65(4):523-527.

15. Miller GE, Freedland KE, Carney RM, et al. Clinical hostility, depressive symptoms, and the expression of inflammatory risk markers for coronary heart disease. J Behav Med. 2003;26(6):501-515.

16. Penninx BWJH, Kritchevsky SB, Yaffe K, et al. Inflammatory markers and depressed mood in Older Persons: Results from the Health, Aging and Body Composition study. Biol Psychiatry. 2003;54(5):566-572.

17. Castanon N, Leonard BE, Neveu PJ, et al. Effects of antidepressants on cytokine production and actions. Brain Behav Immun. 2002;16(5):569574.

18. Dentino AN, Pieper CF, RaoKM, et al. Association of Interleukin- 6 and other biologic variables with Depression in older people living in the community. J Am Geriatr Soc. 1999;47(1):6-11.
19. Steptoe A, Kunz-Ebrecht SR, Owen N. Lack of association between depressive symptoms and markers of immune and vascular inflammation in middle-aged men and women. Psychol med. 2003;33(4):667-674.

20. Kagaya A, Kugaya A, Takebayashi M, et al. Plasma concentrations of Interleukin-1b, Soluble Interleukin-2 Receptor and Tumor Necrosis Factor a of depressed patients in Japan. Neuropsychobiology. 2001;43(2):59-62.

21. Girard D, Tardif J-C, BoisclairDemarble J, et al.Trait Hostility and Acute Inflammatory Responses to Stress in the Laboratory. PLOS ONE. 2016;11(6):e0156329.

22. Boisclair Demarble J, Moskowitz DS, Tardif JC, et al. The relation between hostility and concurrent levels of inflammation is sex, age, and measure dependent. J Psychosom Res. 2014;76(5):384-393.

23. Suarez EC, Lewis JG, Krishnan RR,et al. Enhanced expression of cytokines and chemokines by blood monocytes to in vitro lipopolysaccharide stimulation are associated with hostility and severity of depressive symptoms in healthy women. Psychoneuroendocrinology. 2004;29(9):1119-1128.

24. Larsen JB, Stunes AK, Vaaler A, et al. Cytokines in agitated and nonagitated patients admitted to an acute psychiatric department. PLoS ONE. 2019;14(9):e0222242.

25. Brydon L, Strike PC, Bhattacharyya MR, et al. Hostility and physiological responses to laboratory stress in acute coronary syndrome patients. $J$ Psychosom Res. 2010;68(2):109-116.

26. Suarez EC, Lewis JG, Kuhn C. The relation of aggression, hostility, and anger to Lipopolysaccharide-stimulated TumorNecrosisFactor (TNF)-a by blood monocytes from normal men brain. Behav Immun. 2002;16(6):675-684.

27. Young JS, Bruno D, Pomara N. A review of the relationship between proinflammatory cytokines and major depressive disorder. Journal of Affective Disorders. 2014;169:15-20.

28. Stewart JC, Janicki-Deverts D, Muldoon MF, et al. Depressive symptoms moderate the influence of hostility on serum interleukin-6 and C-reactive protein. Psychosom Med. 2008;70(2):197-204.

29. Graham JE, Robles TF, Kiecolt-Glaser JK, et al. Hostility and pain are related to inflammation in older adults. Brain Behav Immun. 2006;20(4):389-400.

30. Ridker PM, Rifai N, Stampfer MJ, et al. Plasma concentrations of interleukin- 6 and the risk of future myocardial infarction among apparently healthy men. Circulation. 2000;101(15):1767-1772.

31. Mendall M, Patel P, Asante $M$, et al. Relation of serum cytokine concentrations to cardiovascular risk factors and coronary heart disease. Heart. 1997;78(3):273-277.

32. Bertone-Johnson ER, Ronnenberg AG, Houghton SC, et al. Association of inflammation markers with menstrual symptom severity and premenstrual syndrome in young women. Hum Reprod. 2014;29(9):1987-1994.

33. Richards JS. Chapter 1. The Ovarian Cycle. Editors: Gerald Litwack, Vitamins and Hormones. Academic Press. 2018;107:1-25.

34. Syros I, Liapi C, Kolaitis G, et al. Variations of Sex Hormones across the Menstrual Cycle: Correlations to Depressive Symptoms, Hostility, Lipidemic and Haemostatic Factors. J Neuroendocrinol Res. 2018;1(1):101.

35. Syros I, Kolaitis G, Papageorgiou C, et al. Depressive Symptoms do not Affect Lipids and Haemostasis throughout the Menstrual Cycle in Apparently Healthy Young Women. J Neuroendocrinol Res 2018;1(1):103.

36. Fountoulakis KN, lacovides A, Samolis S, et al. Reliability, validity and psychometric properties of the Greek translation of the zung depression rating scale. BMC Psychiatry. 2001;1:6 
37. Zung K, Durham C. A Self-rating depression scale. Arch Gen Psychiatry. 1965;12:63-70.

38. Caine TM, Foulds GA, Hope K. Manual of the hostility and the direction of hostility questionnaire (HDHQ) University of London Press, 1967.

39. Paika V, Almyroudi A, Tomenson B, et al. Personality variables are associated with bowel cancer patients' quality of life independent of psychological distress and disease severity. Psycho-oncology. 2010;19(3):273-282.

40. Felger JC, Lotrich FE. Inflammatory cytokines in depression: neurobiological mechanisms and therapeutic implications. Neuroscience.2013;246:199-229.

41. Yirmiya R, Pollak Y, Morag M, et al. Illness, cytokines, and depression. Ann N YAcadSci. 2000;917:478-487.

42. Himmerich H, Wesemann U, Dalton B, et al. Exploring an association between hostility and serum concentrations of TNF- $\alpha$ and its soluble receptors. J Psychosom Res. 2016;91:87-88.

43. Johnson JD, Barnard DF, Kulp AC, et al. Neuroendocrine Regulation of Brain Cytokines After Psychological Stress. J Endocr Soc. 2019;3(7):13021320

44. Rutledge T, Reis SE, Olson M, et al. Psychosocial variables are associated with Atherosclerosis risk factors among women with chest pain: The Wise Study. Psychosom Med. 2001;63(2):282-288.

45. Suarez EC, Kuhn C, Schanberg S, et al. Neuroendocrine, cardiovascular, and emotional responses of hostile men: the role of interpersonal challenge. Psychosom med. 1998;60(1):78-88
46. Libby P, Ridker PM. Inflammation and atherothrombosis: from population biology and bench research to clinical practice. J Am Coll Cardiol. 2006;48(9):A33-A46.

47. Ross R. Atherosclerosis-an inflammatory disease. $N$ Engl J Med. 1999;14;340(2):115-126.

48. Persons JE, Fiedorowicz JG. Depression and serum low-density lipoprotein: A systematic review and meta-analysis. J Affect Disord. 2016;206:55-67.

49. Davidson KW, Rieckmann N, Rapp MA. Definitions and distinctions among depressive syndromes and symptoms: implications for a better understanding of the depression-cardiovascular disease association. Psychosom Med. 2005;67Suppl 1:S6-S9.

50. Ossewaarde L, Hermans EJ, van Wingen GA, et al. Neural mechanisms underlying changes in stress-sensitivity across the menstrual cycle. Psychoneuroendocrinology. 2010;35(1):47-55.

51. Ossewaarde L, van Wingen GA, Rijpkema M, et al. Menstrual cyclerelated changes in amygdala morphology are associated with changes in stress sensitivity. Hum Brain Mapp. 2013;34(5):1187-1193.

52. Herman JP, Ostrander MM, Mueller NK, et al. Limbic system mechanisms of stress regulation: hypothalamo-pituitary-adrenocortical axis. Prog Neuropsychopharmacol Biol Psychiatry. 2005;29(8):1201-1213. 